\title{
From solar eruption to transformer saturation: the space weather chain
}

\author{
Larisa Trichtchenko \\ Canadian Space Weather Forecast Centre, Natural Resources Canada, 2617 Anderson Rd., \\ Ottawa ON, K1A 0E7, Canada \\ email: larisa.trichtchenko@nrcan-rncan.gc.ca
}

\begin{abstract}
Coronal mass ejections (CME) and associated interplanetary-propagated solar wind disturbances are the established causes of the geomagnetic storms which, in turn, create the most hazardous impacts on power grids. These impacts are due to the large geomagnetically induced currents (GIC) associated with variations of geomagnetic field during storms, which, flowing through the transformer windings, cause extra magnetisation. That can lead to transformer saturation and, in extreme cases, can result in power blackouts. Thus, it is of practical importance to study the solar causes of the large space weather events. This paper presents the example of the space weather chain for the event of 5-6 November 2001 and a table providing complete overview of the largest solar events during solar cycle 23 with their subsequent effects on interplanetary medium and on the ground. This compact overview can be used as guidance for investigations of the solar causes and their predictions, which has a practical importance in everyday life.
\end{abstract}

Keywords. Space Weather, Coronal Mass Ejections, Magnetic Storms, Geomagnetically Induced Currents

The event, presented as a detailed example, started on November 4, 2001 with a halo CME and associated X-ray flare at about 16:20 UT (Fig. 1). Two days later the disturbance arrived at $1 \mathrm{AU}$ as shown in variations of the solar wind magnetic field recorded by the ACE satellite (Fig. 1a). The southward (negative) component reached minimum value of $80 \mathrm{nT}$ at $\sim$ 02:00 UT. On the same day, magnetic field variations in Western Canada (Fig. 1b) maximized at $\sim 04: 00 \mathrm{UT}$ and in Eastern Canada at $\sim$ 02:20 UT (Fig. 1d). Power grids in the West and East (Power site 1 and Power site 2) have responded with GIC maxima at $\sim$ 02:30 UT (site 1) and at $\sim 2: 40$ UT (site 2), as shown in Figs. 1c and 1e.

The complete overview of the most significant Space Weather Events of the Solar Cycle 23 is presented in Table 1 with their subsequent effects on geomagnetic field and power grids. The events were chosen based on the global index of geomagnetic activity $K_{p}>8-$. The halo CME and location (approximate) of the source region, based on location of associated flare, are the key solar parameters. The southward interplanetary magnetic field has a controlling role in the interaction between the ICME and the Earth's magnetosphere. The effects on the ground magnetic field at specific region (eastern Canada) are defined by a peak local hourly range index of magnetic variations. Finally, the peak values of GIC recorded on the Nova Scotia Power system and by the Sunburst monitoring network (Electric Power Research Institute) at one of the power grids in the USA are shown. There were two short power outages during solar cycle 23 attributed to space weather impacts, in New Zealand (November 2001) and in Sweden(October 2003). 
Solar wind parameters
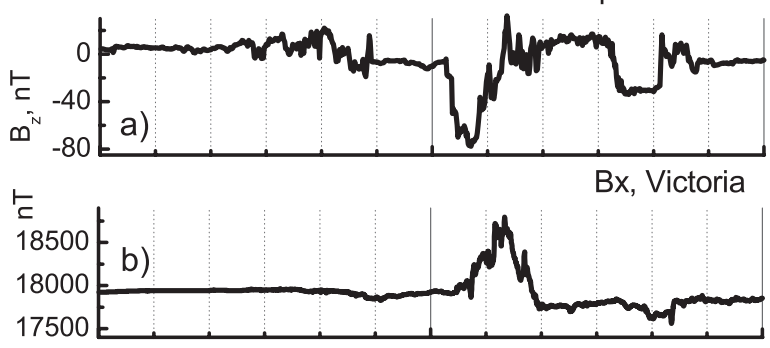

GIC, Power site 1
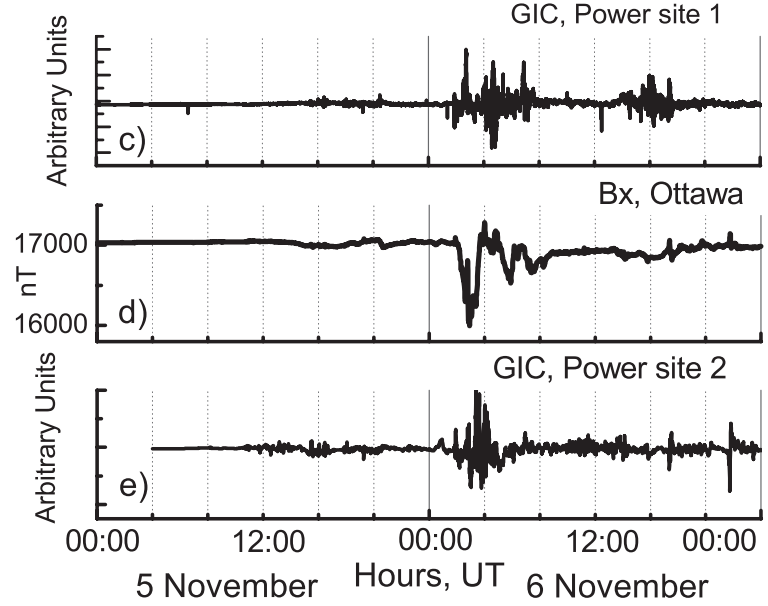

Figure 1. Magnetic field in the solar wind and on the ground and GIC on 5-6 November.

Table 1. Critical parameters of Space Weather Events from the Sun to the Earth.

\begin{tabular}{|c|c|c|c|c|c|c|c|c|}
\hline $\begin{array}{c}\text { Dates } \\
\text { of events }\end{array}$ & CME & $\begin{array}{c}\text { Flare } \\
\text { (assoc.) }\end{array}$ & $\begin{array}{l}\text { Location } \\
\text { (possible) }\end{array}$ & $\begin{array}{c}\mathrm{IMF} \\
B_{z} \\
\mathrm{nT}\end{array}$ & $K_{p}$ & $\begin{array}{c}\text { HR } \\
\text { local } \\
\text { nT }\end{array}$ & $\begin{array}{c}\text { Site } 1 \\
\text { Max } \\
\text { GIC,A }\end{array}$ & $\begin{array}{c}\text { Site } 2 \\
\text { Max } \\
\text { GIC, A }\end{array}$ \\
\hline 2-4 May 98 & Halo & $\mathrm{X} 1, \mathrm{M} 6$ & $\mathrm{~S} 17 \mathrm{~W} 15$ & -32 & 9- & 898 & 70 & 74 \\
\hline 23-25 Sep 98 & $\mathrm{~N} / \mathrm{A}$ & M6.9 & N18E09 & -27 & $8+$ & 1270 & $\mathrm{~N} / \mathrm{A}$ & $\mathrm{N} / \mathrm{A}$ \\
\hline 21-22 Oct 99 & $\mathrm{NE}$ & M1 & $\mathrm{N} / \mathrm{A}$ & -30 & 8 & 908 & 47 & $\mathrm{~N} / \mathrm{A}$ \\
\hline 4-7 Apr 00 & Halo/DSF & $\mathrm{C} 9$ & N16W 66 & -27 & $9-$ & 807 & 96 & 80 \\
\hline 14-16 Jul 00 & Halo & $\mathrm{X} 5$ & $\mathrm{~N} 22 \mathrm{~W} 07$ & -54 & 9 & 1729 & 92 & 76 \\
\hline 17 Sep 00 & Halo & M2 & N13E09 & -34 & $8+$ & 875 & 62 & $\mathrm{~N} / \mathrm{A}$ \\
\hline 31 Mar 01 & Halo & $\mathrm{X} 1.7$ & N20W 19 & -46 & 9 & 1236 & 100 & 76 \\
\hline 11 Apr 01 & Halo & $\mathrm{X} 2$ & S23W 09 & -27 & 8 & 806 & 67 & 27 \\
\hline 06 Nov 01 & Halo & $\mathrm{X} 1$ & N06W 18 & -68 & $9-$ & 1020 & 50 & 64 \\
\hline 24 Nov 01 & Halo & M9.9 & S15W 34 & -40 & 8 & 839 & 67 & 90 \\
\hline 23 May 02 & Halo/DSF & C5 LDF & S $22 \mathrm{~W} 53$ & -43 & $8+$ & 327 & 24 & 10 \\
\hline 29-30 May 03 & $? ?$ & X1, X3 & S07W 17 & -33 & $8+$ & 915 & 40 & 60 \\
\hline $29-31$ Oct 03 & 2Halo & $\mathrm{X} 17 / \mathrm{X} 10$ & $\begin{array}{l}\text { S16E08/ } \\
\text { S15W } 02\end{array}$ & $\begin{array}{l}-48 / \\
-35\end{array}$ & 9 & 1500 & 100 & 75 \\
\hline $20-21$ Nov 03 & Halo & M3 & S01E18(?) & -53 & $9-$ & 450 & 60 & 20 \\
\hline 24-27 Jul 04 & Halo & $\mathrm{M} 1.2 \mathrm{LDE}$ & N03W 27 & -21 & $9-$ & 1422 & $\mathrm{~N} / \mathrm{A}$ & 50 \\
\hline 7-8 Nov 04 & Halo & M5/M9 & $\begin{array}{c}\text { N11E11/ } \\
\text { N10E08 }\end{array}$ & -48.5 & 9- & 1561 & $\mathrm{~N} / \mathrm{A}$ & 78 \\
\hline $9-10$ Nov 04 & Halo & X2/M9 & $\begin{array}{l}\text { N09W 17/ } \\
\text { N07W } 51\end{array}$ & -25 & $9-$ & 1317 & $\mathrm{~N} / \mathrm{A}$ & 80 \\
\hline 15 May 05 & Halo & M8 & N12E12 & -43 & $8-$ & 1924 & $\mathrm{~N} / \mathrm{A}$ & 83 \\
\hline 24 Aug 05 & Halo & $\begin{array}{c}\mathrm{M} 2 / \mathrm{M} 5 \\
\mathrm{LDE}\end{array}$ & $\begin{array}{c}\text { S08W 50/ } \\
\text { S12W } 60\end{array}$ & -55 & $9-$ & 662 & $\mathrm{~N} / \mathrm{A}$ & 55 \\
\hline
\end{tabular}

$\mathrm{DSF}=$ Disappearing Solar Filament, LDE=Long Duration Event, N/A=data not available 